\title{
LEER LA SUPERFICIE: LA PRESENCIA DE GILLES DELEUZE EN LA ERA POSTEÓRICA DE LA CRÍTICA LITERARIA
}

\section{David PRUNEDA SENTÍES}

Universidad Nacional Autónoma de México prunedasenties@gmail.com

diferencia de los estudios literarios de las décadas de 1980 y 1990, la era posteórica de la
crítica literaria presenta una apropiación más completa de las ideas de Gilles Deleuze.
Mientras que los primeros usos de sus conceptos pueden considerarse aplicaciones de herramientas teóricas, en la actualidad existen prácticas de lectura que se apuntalan sobre postulados del filósofo francés. Aunque este no es el único respaldo de estas prácticas de lectura y en ocasiones ni siquiera reconocen la deuda que tienen con las ideas de Deleuze, su presencia es evidente. De hecho, la omisión de esta influencia es una consecuencia de los procesos de asimilación e institucionalización de la obra deleuziana en la crítica literaria.

Antes que nada, es necesario hacer una descripción sucinta del proceso de recepción en los Estados Unidos de América de las ideas filosóficas francesas de las décadas de 1960 y 1970. El propósito de esta revisión es dar una panorámica de la inserción de autores como Derrida, Foucault y Deleuze en la academia estadounidense, creadora de la hoy llamada "Teoría francesa" en los estudios literarios. En su análisis de este fenómeno, François Cusset (2003: 1) equipara a estos intelectuales con las estrellas del Western hollywoodense: Derrida es Clint Eastwood; Baudrillard, Gregory Peck; Foucault, Steve McQueen; Kristeva, Meryl Streep y Cixous, Faye Dunaway. En cuanto a Deleuze y Guattari, sus homólogos cinematográficos son Paul Newman y Robert Redford en Butch Cassidy and the Sundance Kid. Si bien el tono de la comparación es francamente paródico, el propósito de este ejercicio es ilustrar el grado de fama que alcanzaron estos pensadores en la esfera intelectual y en la cultura popular de E.U.A. Puesto que sus ideas se permearon hacia ámbitos como la música electrónica, la Internet, el arte conceptual, el cine comercial y, sobre todo, las discusiones en torno a la cultura y la política, Cusset (2003: 2) afirma que ni siquiera en Francia estos autores consiguieron el nivel de notoriedad e influencia logrados en E.U.A. La principal causa de este éxito masivo se fraguó, curiosamente, en las universidades. 
La Teoría francesa en los estudios literarios es el resultado de una cierta violencia taxonómica ejercida por la academia estadounidense. Los factores que dieron pie a este movimiento tienen raíces sociales, ya que los intelectuales de izquierda de los ochenta que buscaban configurar un nuevo discurso político encontraron sus herramientas en los textos de los pensadores franceses. De esta manera dio inicio un proceso de apropiación conceptual mediante una serie de operaciones. La primera consistió en reunir las obras en un corpus homogéneo, que permitió el manejo de un vocabulario común y que funcionó, al mismo tiempo, como una fuente de complicidad entre sus usuarios (Cusset, 2003: 8). Sobra decir que semejante maniobra requirió de innumerables negociaciones —en el mejor de los casos - y una buena cantidad de omisiones - en el peor. Sin importar las evidentes discrepancias intere intraproyectos teóricos, la Teoría francesa fue el saco en el que selectivamente se echaron los postulados producidos en Francia durante los últimos veinte años. Las siguientes operaciones de la academia estadounidense fueron las de etiquetado y "branding", así como la reorganización de conceptos y su redistribución en el ámbito práctico (Cusset, 2003: 10). Es justo decir que este proceso tuvo por lo menos dos consecuencias sumamente productivas. En primer lugar, ciertos textos que habían quedado relegados en la academia francesa o encasillados en la esfera editorial vivieron una reactivación a partir de relecturas e interpretaciones críticas. En segundo, tanto la aglutinación como la digestión de este contenido teórico facilitaron y promovieron su uso político en la esfera pública estadounidense. Hacia finales de la década de 1980, los estudios literarios en E.U.A. no solo se armaron de un arsenal conceptual, sino que también se convirtieron en los proveedores de herramientas discursivas con impacto social.

No es muy aventurado decir que los departamentos de literatura fueron los principales responsables de la fama de la Teoría francesa en la cultura estadounidense. Si bien resulta llamativo el hecho de que hayan sido los críticos literarios quienes introdujeron en el currículo universitario ideas que tuvieron su origen en otras disciplinas, hay dos factores que lo explican. Por un lado, la academia literaria de E.U.A. ya había manifestado un hambre insaciable por los instrumentos de análisis; por el otro, desde mediados de los setenta, el contexto universitario presenció una pugna entre las diferentes disciplinas para conseguir la hegemonía de los campos del conocimiento. Los estudios literarios habrían de salir victoriosos de este conflicto (Cusset, 2003: 78), convirtiéndose en el filtro mediante el cual la academia estadounidense procesó y reconfiguró los contenidos teóricos franceses, tanto para sus propios fines como para su relanzamiento internacional.

Es verdad que cuando se trata de nuevos acercamientos o nuevas metodologías, toda disciplina humanística busca llevar agua a su molino. En ese sentido los estudios literarios en E.U.A. no fueron la excepción. Los primeros usos prácticos de la Teoría francesa consistieron en la extrapolación directa — en la medida de lo posible - de los conceptos teóricos al análisis literario. De un día para otro tuvo lugar una profusión de artículos que presentaban una revisión del vínculo entre Foucault y la literatura, por ejemplo, o una lista de las ideas foucaultianas más útiles para la crítica de textos. Esto mismo sucedió con otros teóricos franceses. Deleuze y Guattari, de manera un tanto retardada, también 
experimentarían este destino (Cusset, 2003: 79-80). En su caso, el concepto de "literatura menor" fue uno de los primeros en ser acarreados al molino de la crítica literaria.

De acuerdo con la definición de Deleuze y Guattari (1975: 28), una «literatura menor no es la literatura de un idioma menor, sino la literatura que una minoría hace dentro de una lengua mayor». Es decir, la existencia de una literatura menor depende, en primer lugar, de un uso particular de la lengua. Antes que a un grupo social minoritario, los escritores menores pertenecen a una minoría puesto que desterritorializan un idioma mayor (Deleuze \& Guattari, 1975: 37). La literatura producida por una minoría en el sentido social no necesariamente es una literatura menor porque provenga de grupos marginados. Estas minorías solo pueden considerarse como tales, literariamente hablando, cuando realicen una marcada desterritorialización de la lengua mayor en la que se expresan. En otras palabras, la condición menor de una literatura no depende de su origen sociodemográfico, sino de su forma de expresión discursiva.

A raíz de un congreso llevado a cabo en 1986 en Berkley, California, Abdul JanMohamed y David Lloyd inauguraron una corriente crítica dedicada a las literaturas menores de E.U.A. Los resultados se publicaron en un extenso libro que incluye análisis sobre la estética y la estilística de las minorías estadounidenses, como la afroamericana, la irlandesa y la amerindia. Por supuesto, los ensayos se encuentran en clave de las ideas de Deleuze y Guattari. Probablemente esta compilación contenga uno de los usos más cuidadosos que se le han dado al concepto de literatura menor, por ejemplo, el texto de Lloyd (1990: 370) inicia preguntándose cuál es la relación histórica entre una literatura menor y una literatura de minorías. Esta distinción importantísima habla acerca de la cautela que debe tenerse cuando se utilizan las ideas de la Teoría francesa en la discusión crítica de textos literarios. Un caso contrario es el de Roland Bogue (2012: 199), quien comete quizás el error más común en este asunto e identifica la literatura menor con la literatura producida por minorías.

La discrepancia entre estas dos interpretaciones puede deberse a una cuestión de contexto. Puesto que Lloyd escribe durante la bonanza teórica de la década de 1980, su lectura de Deleuze y Guattari es más meticulosa. No solo porque los textos, en su calidad de novedosos, requerían de una mayor atención para su procesamiento académico, sino también porque el debate universitario se encontraba en pleno auge, motivando un mayor rigor en la práctica crítica. El artículo de Bogue, por su parte, se publicó en el 2012 y es probable que la distancia temporal le haya jugado una mala pasada. Tanto se ha escrito sobre la literatura menor de Deleuze y Guattari que un exceso de interpretaciones puede fácilmente conducir a malos entendidos. No obstante, el caso de Bogue es un ejemplo de la reactivación conceptual mencionada anteriormente, en donde la malinterpretación de un texto puede ser incluso más productiva y esclarecedora que su lectura fiel. Lloyd, por su parte, tampoco está exento. Si bien es muy cuidadoso en no equiparar a la minoría con la literatura menor, su enumeración de las características del concepto de Deleuze y Guattari solo podría definirse como una paráfrasis bastante libre (cfr. Lloyd, 1990: 381).

¿Por qué estas descripciones son tan diferentes de la que se presenta en el libro Kafka. Por una literatura menor? Una de las principales razones es la extrapolación de conceptos de una disciplina a 
otra. Definitivamente, este fue un fenómeno muy común en la academia estadounidense en las décadas de los ochenta y los noventa; sin embargo, algo similar sucede incluso treinta años después. Lo cierto es que no importa qué tan cuidadoso sea el crítico literario con los conceptos que utiliza, debe enfrentar esta dificultad cada vez que se propone echar mano de ideas que provengan de la teoría en general y de la Teoría francesa en particular. No sorprende, pues, que en los primeros años del siglo XXI la pertinencia de la teoría en los estudios literarios se pusiera en duda, dando lugar a lo que algunos denominaron la era posteórica de los estudios literarios.

Aunque se piense que este cuestionamiento desembocaría en un abandono de los contenidos teóricos para la práctica crítica, la naturaleza de la era posteórica de la academia literaria es muy diferente. Jean-Michel Rabaté (2002: 4) declara que la defunción de la teoría ha sido anunciada en repetidas ocasiones, pero que en ningún otro momento de la historia ha habido tantas guías, antologías, ediciones críticas, simposios, congresos y recapitulaciones metodológicas en torno a los conceptos de la Teoría francesa. Por un lado, para algunos de los autores que durante la primera década del nuevo milenio publicaron libros sobre un cambio en el rumbo de los estudios literarios, como Terry Eagleton, la respuesta a la crisis es la producción de más teoría. Por otro lado, hay críticos que asumen una postura diferente, aunque no necesariamente contraria. Valentine Cunningham (2002: 168-9), por ejemplo, acepta que en la actualidad todos llevamos algún tipo de teoría a nuestra práctica de lectura, pero que es necesario abogar por «un respeto racional, correcto e incluso moral de la primacía del texto por encima de la teorización sobre el texto» (La traducción es mía). Cierta facción de la academia estadounidense contemporánea es más afín a las ideas de Cunningham que a las de Eagleton.

Una fuente seminal para esta tendencia es Susan Sontag, quien arremete contra la lectura fundamentada en el modelo interpretativo de Freud y Marx, dos de los pilares de la Teoría francesa. De acuerdo con esta autora, el intérprete que utiliza este modelo altera el significado verdadero del texto en lugar de descifrarlo. Sontag considera que este es un acto de violencia, por lo que propone que el crítico no debe exponer un significado oculto, sino que debe mostrar la obra tal como es (Marcus \& Best, 2009: 10). Sobre la misma línea se encuentra Eve Kosofsky Sedgwick (2002: 130), quien afirma que el uso de ciertas teorías para la interpretación de textos literarios conduce a prácticas paranoicas de lectura y argumenta que la principal razón para cuestionarlas es la posibilidad de que sus sospechas puedan ser ilusorias o simplemente incorrectas. De esta manera, ambas autoras atacan una estrategia de análisis denominada lectura sintomática, cuyo propósito es destapar la problemática oculta de un texto (Thurston, 1993: 638). En la era posteórica de la academia estadounidense, este embate sería retomado por Sharon Marcus y Stephen Best en The Way We Read Now, un número especial de la revista Representations, publicada por la Universidad de California.

Marcus y Best exponen sus reservas para con la lectura sintomática en el texto introductorio. Critican el hecho de que esta práctica de lectura argumente que lo más interesante de un texto es aquello que reprime, omite o esconde. Es labor del crítico, entonces, detectarlo mediante un grado extremo de perspicacia y entendimiento (Marcus \& Best, 2009: 3-4). El problema radica en que la lectura sintomática pone énfasis en el intérprete y no en la obra interpretada, puesto que presenta al crítico 
literario como una suerte de héroe capaz de desentrañar los mensajes ocultos de sus objetos de estudio. No obstante, el reclamo más fuerte de los editores se dirige a que la lectura sintomática da por sentado que el texto es la superficie de una profundidad. Esta suposición involucra una serie de connotaciones negativas, por ejemplo que «la superficie está asociada con lo superficial y lo engañoso, con lo que puede advertirse sin un análisis detallado y que, implícitamente, podría resultar falso bajo un escrutinio más cuidadoso» (Marcus \& Best, 2009: 4. La traducción es mía). Por un lado, el desacuerdo con estas ideas es lo que motiva a Marcus y a Best a redimir el concepto de superficie en la crítica literaria. Por el otro, a pesar de que su intención haya sido alejarse de la Teoría francesa y de sus pilares, esta maniobra acerca peligrosamente a los editores a las nociones que propone Deleuze en Lógica del sentido. De hecho, considerando que es uno de los productos de una academia estadounidense altamente influenciada por la Teoría francesa, es posible decir que la lectura de superficie es, en esencia, una práctica deleuziana.

La propuesta de Marcus y Best bien podría sintetizarse en una aseveración de Deleuze: «si no hay nada que ver detrás del telón es que todo lo visible, o más bien toda la ciencia posible está a lo largo del telón» (1969: 36). Los editores de The Way We Read Now están interesados en resignificar la noción de superficie no como una capa exterior que oculta un significado profundo, sino como algo que es

[...] evidente, perceptible, aprehensible en los textos; algo que no oculta ni que está oculto; algo que, en el sentido geométrico, tiene longitud y anchura pero no grosor, y que, por tanto, no cubre una profundidad. Una superficie es algo que insiste en ser visto y no algo a través de lo que tenemos que ver. (Marcus \& Best, 2009: 9; la traducción es mía)

Los textos muestran sus propias verdades porque se autorregulan, porque todo lo que supuestamente aporta la teoría ya se encuentra presente en la obra desde el principio. Por lo tanto, la práctica del crítico no consiste en descifrar, sino en describir:

El propósito de la crítica es entonces relativamente modesto: indicar lo que el texto dice sobre sí mismo. Aquí la profundidad no se encuentra fuera del texto o debajo de su superficie [...] más bien, la profundidad forma parte de un continuo con la superficie y es, así, un efecto de inmanencia. (Marcus \& Best, 2009: 11; la traducción es mía)

No hay duda de que la última oración de la cita anterior resuena significativamente tanto con el léxico de Deleuze - una prueba más del grado de asimilación de la Teoría francesa en la academiacomo con su concepto de acontecimiento. «Los acontecimientos, en su diferencia radical con las cosas, ya no son buscados en profundidad, sino en la superficie, en este tenue vapor incorporal que se escapa de los cuerpos, película sin volumen que los rodea, espejo que los refleja, tablero que los planifica» (Deleuze, 2005: 36). Así, desde esta perspectiva, la superficie no es una instancia más del texto, es el texto mismo.

Para continuar con la comparación entre la lectura de superficie y las ideas de Deleuze - puesto que uno tiene que llevar agua a su molino - es inevitable arriesgar un cotejo de nociones. La esencia deleuziana de la propuesta de Marcus y Best se vuelve más clara cuando se traza una correspondencia 
entre el cuerpo o la cosa en Deleuze y el texto como objeto material. De acuerdo con los editores de The Way We Read Now, la superficie debe considerarse una materialidad (Marcus \& Best, 2009: 9). Esta concepción no solo pone de manifiesto la necesidad de un soporte tangible, sino también su papel en el proceso de significación. Es mediante el acto de lectura de la materialidad que la crítica accede al significado perceptible de la obra. El acto de lectura en la propuesta de Marcus y Best se vuelve un acontecimiento en su acepción deleuziana, ya que cuando un sujeto lee un texto se produce un nuevo atributo en la superficie del objeto material, el de ser leído. (Esto, por supuesto, está en consonancia con el famoso ejemplo de Deleuze del árbol que verdea). Ahora bien, no es posible hablar de acontecimiento sin hablar del sentido:

En una palabra, el sentido es siempre un efecto. No solamente un efecto en el sentido causal, sino un efecto en el sentido de «efecto óptico», «efecto sonoro» o, mejor aún, efecto de superficie, efecto de posición, efecto del lenguaje. Un efecto semejante no es en absoluto una apariencia o una ilusión; es un producto que se extiende o se alarga en la superficie, y que es estrictamente copresente, coextensivo a su propia causa, y que determina esta causa como causa inmanente, inseparable de sus efectos. (Deleuze, 1969: 101)

Desde la perspectiva de la crítica literaria, el acontecimiento de la lectura sobre el cuerpo del texto produce el sentido como un efecto de superficie. Asimismo, si el sentido es copresente a su propia causa, entonces la superficie de un texto leído ya contiene su propio sentido desde el principio. Es por esto que Marcus y Best (2009: 18) argumentan que todo lo que es digno de nuestra atención en un texto literario ya se encuentra a simple vista en la superficie de la obra.

Por último, merece la pena señalar una coincidencia más entre la propuesta crítica en The Way We Read Now y el texto de Lógica del sentido. Este paralelismo bien podría tacharse de involuntario e, incluso, desafortunado para las expectativas de Marcus y Best. Así como el texto de Deleuze está organizado en series de paradojas, podría decirse que la lectura de superficie está aquejada por dos de estas. La primera radica en la práctica de los estudios literarios. Como ya se mencionó, los editores afirman que más que interpretar, el crítico debe describir lo que el texto ya expresa por sí mismo. Esto acarrea ciertas dificultades, en especial si se tiene en cuenta que cualquier descripción involucra necesariamente una interpretación. Es desde este punto débil que Ellen Rooney emprende un ataque frontal a la lectura de superficie. De acuerdo con la académica, el hecho de que Marcus y Best afirmen que la lectura de superficie limita el acto crítico a la "modesta" práctica de la descripción y que, por lo tanto, el énfasis recae en la obra estudiada y no en el sujeto que la estudia, es una estrategia en extremo soberbia. La lectura de superficie, según Rooney (2010: 125), provoca el resultado opuesto, ya que distraídamente, quizá - el crítico se inviste de la autoridad para definir qué es lo que el texto revela por sí mismo y cierra, de manera un tanto autoritaria, la posibilidad de estudios posteriores con direcciones distintas.

La segunda paradoja ya se ha mencionado y tiene que ver con el propósito explícito de los editores de alejarse de una crítica literaria construida con bases teóricas. Como se demostró en este breve análisis, la lectura de superficie tiene las raíces fuertemente amarradas a la Teoría francesa en general y a determinados conceptos de Deleuze en particular. Es probable que esta propuesta de lectura contenga fundamentos que provengan de otras tradiciones teóricas y que también hayan pasado 
desapercibidos para Marcus y para Best. Lo cierto es que en la actualidad, el uso de teoría en los estudios literarios no se confina a la extrapolación de nociones e ideas, sino que la marcada asimilación de las mismas ha contribuido al levantamiento de prácticas críticas enteras, como la lectura distante, la lectura cognitiva, la lectura obbligato y la lectura reconstructiva, entre otras.

\section{Referencias bibliográficas}

Bogue, R. (2012): «Deleuze and Literature», en D. W. SMith y H. Somers-HaLl, eds., The Cambridge Companion to Deleuze, Cambridge, Cambridge University Press, pp. 286-306.

Cunningham, V. (2002): Reading After Theory, Oxford, Blackwell.

Cusset, F. (2003): French Theory. How Foucault, Derrida, Deleuze, \& Co. Transformed the Intellectual Life of the United States, J. Fort, trad., Minneapolis, University of Minnesota Press, 2008.

DeleuZe, G. (1969): Lógica del sentido, M. Morey, trad., Barcelona, Paidós, 2005.

Deleuze, Gilles - GuAtTARI, F. (1975): Kafka. Por una literatura menor, J. Aguilar Mora, trad., México, Era, 1990.

EAgleton, T. (2003): After Theory, Nueva York, Basic Books.

JanMohamed, A. - LloYd, D., eds. (1990): The Nature and Context of Minority Discourse, Nueva York, Oxford University Press.

MARCus, S. - Best, S., eds. (2009): The Way We Read Now. Num. esp. Representations, en http://www.jstor.org/stable/10.1525/rep.2009.108.1.toc (última consulta, 27-2-2015).

RABATÉ, J.-M. (2002): The Future of Theory, Oxford, Blackwell.

RoONEY, E. (2010): «Live Free or Die: The Reading Effect and the Persistence of Form», differences, en https://fadingtheaesthetic.files.wordpress.com/2014/08/live-free-or-describe.pdf (última consulta, 15-02-2015).

SEDGwiCK, E. K. (2003): «Paranoid Reading and Reparative Reading, or, You're so Paranoid, You Probably Think this Essay is About You», en Touching Feeling: Affect, Pedagogy, Performativity, Durham, N. C., Duke University Press, pp. 123-151.

SONTAG, S. (1966): «Against Interpretation», en Against Interpretation, and Other Essays, Farrar, Nueva York, Strauss and Giroux, pp. 3-14.

ThURSTON, J. (1993): «Symptomatic Reading», en I. R. MAKARYK, ed. y comp., Encyclopedia of Contemporary Literary Theory: Approaches, Scholars, Terms, , Toronto, University of Toronto Press, p. 638. 